\title{
Effect of Adenosine-induced Changes in Presynaptic Release Probability on Long-Term Potentiation in the Hippocampal CA1 Region
}

\author{
F. Asztely, ${ }^{1}$ M.-Y. Xiao, ${ }^{2}$, H. Wigström, ${ }^{2}$ and B. Gustafsson ${ }^{1}$ \\ 'Department of Physiology and ${ }^{2}$ Department of Medical Physics, University of Göteborg, Göteborg, Sweden
}

In the present study some characteristics of long-term potentiation (LTP) in the hippocampal CA1 region were examined under different conditions of transmitter release. Adenosine A1 agonist/antagonists, or in some instances changes in the extracellular calcium/magnesium ratio, were used to alter release probability. The overall LTP time course (onset latency, growth phase, and subsequent decay for both the non-NMDA and NMDA receptor-mediated EPSPs) following a brief tetanus was essentially the same over an almost 10 -fold variation in release probability (measured as change in field EPSP magnitude). The major difference observed was a faster initial decay of LTP evoked at low levels of release probability, possibly related to impaired induction conditions. It was also observed that LTP induced at one level of release probability occluded that induced at a lower (or higher) level, and that changes in release probability induced by adenosine agonist/antagonists affected potentiated and "naive" EPSPs to an equal extent. Taken together, these data do not provide support for the notion of different locations for LTP expression at different conditions of release probability. The results are also more compatible with the notion of a single, rather than several, expression mechanism(s) within the first hour of LTP in the hippocampal CA1 region.

[Key words: hippocampus, long-term potentiation, transmitter release, release probability, adenosine, paired pulse facilitation]

Long-term potentiation (LTP) is a form of synaptic plasticity thought to be a cellular correlate of learning and memory (Bliss and Collingridge, 1993). In the hippocampal CA1 region there is now considerable evidence for a postsynaptic induction of LTP, but whether LTP is expressed pre- or postsynaptically is still uncertain. An interesting possibility, recently suggested, is that LTP is expressed at both locations (Kullman and Nicoll, 1992; Larkman et al., 1992; Liao et al., 1992) and that the degree to which LTP is expressed pre- or postsynaptically is determined by the initial conditions of synaptic release probability (Larkman et al., 1992; Liao et al., 1992). For example, under conditions of high release probability, obtained by an increase in

\footnotetext{
Received Dec. 20, 1993; revised May 2, 1994; accepted May 5, 1994.

This work was supported by the Swedish Medical Research Council (Projects 05180 and 05954); the foundations of Magnus Bergvall, Åke Wiberg and O. E. and Edla Johansson, Svenska Sällskapet för Medicinsk Forskning; and The Royal Society of Arts and Sciences in Gothenburg.

Correspondence should be addressed to Fredrik Asztely, Department of Physiology, University of Göteborg, Medicinaregatan 13, S-413 90 Göteborg, Sweden. Copyright (C) 1994 Society for Neuroscience $0270-6474 / 94 / 146707-08 \$ 05.00 / 0$
}

the extracellular concentration of calcium ions, LTP is expressed mainly postsynaptically (Larkman et al., 1992). As suggested by Hannay et al. (1993) the initial conditions of release probability, per se, may determine the locus of LTP expression by influencing the combination of second messenger systems triggered.

When evoked by a brief tetanus LTP in the CA1 region describes an early complex time course before settling at a more stable level (Gustafsson et al., 1989). Thus, following the induction event there is a latency oî a few seconds before LTP appears, and a growth phase during $20-40 \mathrm{sec}$ that is followed by a decay that may last for tens of minutes. Since the increase in postsynaptic calcium that triggers LTP only needs to be very brief (less than 2-2.5 sec, Malenka et al., 1992) there is no direct link between the increased intracellular levels of calcium and the early growth and decay of LTP. Thus, the temporal development of LTP seems to be governed by the temporal characteristics either of second messenger systems activated by calcium or of the mechanism(s) underlying the expression of LTP. Since these systems and/or mechanisms might well differ for a pre- and postsynaptic location of LTP, one might expect a variation in the temporal characteristics of LTP dependent on its location. The aim of the present study is to examine these possibilities by studying the temporal development of LTP under different conditions of initial release probability.

\section{Materials and Methods}

Experiments were performed on hippocampal slices from 54 adult guinea pigs, weighing $250-400 \mathrm{gm}$. The slices were prepared as previously described (Gustafsson et al., 1989) and were maintained in a constant flow incubation chamber (Brain Slice Chamber, Medical Systems Corp., Greenvale, NY), half-submerged in an oxygenated solution containing (in $\mathrm{mm}$ ) $\mathrm{NaCl}, 124 ; \mathrm{KCl}, 4 ; \mathrm{CaCl}_{2}, 4 ; \mathrm{MgCl}_{2}, 4 ; \mathrm{NaHCO}_{3}, 26 ; \mathrm{NaH}_{2} \mathrm{PO}_{4}$, 1.25; glucose, 10 ; and gassed with $95 \% \mathrm{O}_{2}, 5 \% \mathrm{CO}_{2}$. All experiments were performed in the presence of $100 \mu \mathrm{M}$ picrotoxin in order to block fast GABAergic inhibitory postsynaptic potentials (IPSPs). The higher than normal concentrations of calcium and magnesium were used to reduce the excitability increase during this blockade of inhibition. To obtain a pure NMDA receptor-mediated field excitatory postsynaptic potential (EPSP), or a combined NMDA- and non-NMDA receptormediated EPSP (cf. Asztely et al., 1992), the perfusion fluid contained (in $\mathrm{mM}$ ) $\mathrm{NaCl}, 124 ; \mathrm{KCl}, 4 ; \mathrm{CaCl}_{2}, 2 ; \mathrm{MgCl}_{2}, 0.1 ; \mathrm{NaHCO}_{3}, 26 ; \mathrm{Na}$ $\mathrm{H}_{2} \mathrm{PO}_{4}, 1.25$; glucose, 10; and 6-cyano-7-nitroquinoxaline-2,3-dione (CNQX), $10 \mu \mathrm{M}$ (pure NMDA receptor-mediated EPSPs) or $1.0 \mu \mathrm{M}$ (combined NMDA- and non-NMDA-receptor-mediated EPSPs). The temperature in the recording chamber was maintained at $30^{\circ} \mathrm{C}$. A surgical cut was made between the CA3 and CA1 regions to prevent epileptiform bursting in CA3 cells from affecting the recordings in CA1 (cf. Thompson et al., 1992). It will also prevent the reduction of the presynaptic fiber potentials due to hyperpolarization of Schaffer collaterals by adenosine agonists (Dunwiddie and Miller, 1993).

Electrical stimulation of afferent fibers and recordings of extracellular synaptic field potentials were carried out in the apical dendritic layer 
Table 1. Augmentation in "control" solution in the presence of CHA and in the presence of DPCPX, respectively

\begin{tabular}{llll} 
& CHA & "Control" & DPCPX \\
\hline Peak value & $63.0 \pm 12.8 \%$ & $61.2 \pm 8.6 \%$ & $38.8 \pm 5.2 \%$ \\
& $n=35$ & $n=14$ & $n=32$ \\
Decay constant & $8.9 \mathrm{sec}$ & $7.9 \mathrm{sec}$ & $9.2 \mathrm{sec}$
\end{tabular}

(stratum radiatum) of the CAl pyramidal cells. Stimuli consisted of 0.1 msec negative constant-current pulses $(20-80 \mu \mathrm{A})$ delivered through electrolytically sharpened tungsten wires (monopolar stimulation). Two independent inputs were always used, the two stimulus electrodes positioned on either side of the recording electrode (Wigström and Gustafsson, 1985). Both inputs received a test stimulus every $4 \mathrm{sec}$, but 2 sec apart. For tetanic stimulation, high-frequency stimulation of $50 \mathrm{~Hz}$ was always used. To saturate LTP the input was subjected to five 20 impulse tetani, $30 \mathrm{sec}$ apart, using $1-1.5 \times$ test strength, repeated three or four times with 5-10 min interval. Recordings were made by means of glass micropipettes filled with $3 \mathrm{M} \mathrm{NaCl}$ (1.5-3 M $\Omega$ resistance). Field potentials were amplified with an Axoclamp-2A (Axon Instruments, Foster City, CA), filtered at $3 \mathrm{kHz}$ and recorded on line using a Nord10 microcomputer. The slope of the rising phase of the EPSP was measured by means of linear regression of the first $0.75-1.0$ msec succeeding the presynaptic volley. Paired pulse facilitation (cf. Zucker, 1989) was always tested using an interstimulus interval of $50 \mathrm{msec}$, and was calculated as the percentage increase of the initial slope of the second EPSP compared to that of the first. Values are given as mean \pm SEM.

Selective agonists and antagonists to the adenosine Al receptor were used to change the release probability (Lupica et al., 1992; Prince and Stevens, 1992; Scholz and Miller, 1992). The A1 reccptor agonist $N^{6}-$ cyclohexyladenosine (CHA; 100-200 nM) changed paired pulse facilitation from $28 \pm 7.9 \%$ to $143 \pm 18.9 \%, n=10$, and decreased the field EPSP to $20 \pm 2.8 \%$ of its original value, whereas the antagonist 8-cyclopentyl-1,3-dipropylxanthine (DPCPX; $200 \mathrm{nM}$ ) gave rise to a paired pulse depression of $5.4 \pm 2.7 \%(n=4)$ and increased the field EPSP by $60 \pm 14 \%(n=5)$. These changes in EPSP magnitude were not associated with observable changes in the presynaptic volley. Following the application of CHA and DPCPX, respectively, the stimulus strength was adjusted to obtain EPSPs of similar magnitude as before the application of the drugs.

Following a 10 impulse tetanus, presynaptic aftereffects, unrelated to LTP, decay within 20-30 sec (McNaughton, 1982; Gustafsson et al., 1989), and thus reflect augmentation (Magleby and Zengel, 1976; Zucker, 1989). To obtain the augmentation in isolation tetani were evoked in the presence of the NMDA-receptor antagonist $\mathrm{D}(-)$-2-amino-5phosphonopentanoic acid (D-AP5; $50 \mu \mathrm{M})$. The decay time constant of augmentation was found to be practically unaltered by application of adenosine Al agonist/antagonists, although the peak value was found to be decreased in the presence of DPCPX (see Table 1). To obtain the early time course of LTP exclusive of augmentation, the original potentiation values were divided by the averaged augmentation curve obtained in presence of the respective agonist/antagonist.

Drugs. D(-)-2-Amino-5-phosphonopentanoic acid (D-AP5) and 6-cyano-7-nitroquinoxaline-2,3-dione (CNQX) were obtained from Tocris Neuramin, UK; picrotoxin and $N^{6}$-cyclohexyladenosine (CHA) were from Sigma Chemical Company, USA; and 8-cyclopentyl-1,3-dipropylxanthine (DPCPX) was from Research Biochemicals International, USA.

\section{Results}

\section{Effect of adenosine agonist/antagonists on field EPSP magnitude}

Application of the adenosine Al receptor agonist CHA (100$200 \mathrm{nM})$ decreased the field EPSP to $20 \pm 2.8 \%(n=10)$ of its original value, indicating a decrease of the release probability. In some of the experiments one of the two inputs was repeatedly tetanized to saturate for LTP (see Materials and Methods) before drug application, causing a $110-230 \%$ (mean value $=150 \%$ ) potentiation. The subsequent application of $\mathrm{CHA}$ reduced both inputs to an equal degree (paired difference of $0.4 \pm 7.3 \%, n=$
A

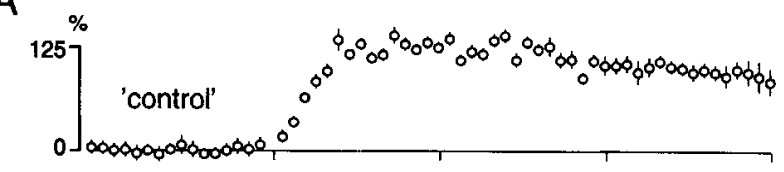

B

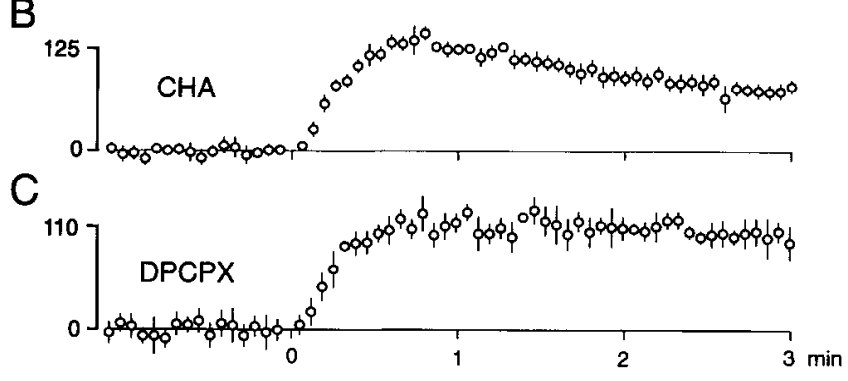

Figure 1. LTP growth phase following a 10 impulse tetanus $(50 \mathrm{~Hz})$ in different conditions of release probability. $A$, Average EPSP changes $( \pm$ SEM), as percentage of the average EPSP value obtained during the $60 \mathrm{sec}$ immediately preceding the tetanus, in "control" solution (i.e., no adenosine agonist/antagonists present) $(n=34)$. To obtain the early time course exclusive of augmentation, the original potentiation values were divided by the averaged augmentation curve obtained in the same experiments in the presence of D-AP5. In the figure the potentiations are normalized to a common peak amplitude (note the different scales of the ordinates). $B$, Same as in $A$, but in the presence of 100-200 nM CHA $(n=24)$. C, Same as in $A$, but in the presence of $200 \mathrm{~nm} \mathrm{DPCPX}$ $(n=10)$.

5). When subsequently tetanized (a single 10 impulse tetanus) in the presence of CHA no significant potentiation ( $-0.3 \%$ ) was observed in the previously tetanized inputs compared to a $33 \%$ potentiation in the naive ones (measured $10 \mathrm{~min}$ after the tetanus, $n=5$ ), that is, indicating occlusion between LTPs evoked at these different conditions of release probability.

Application of the adenosine Al receptor antagonist DPCPX (200 nM) increased the field EPSP by $60 \pm 14 \%(n=5)$. As in the case of CHA, in some of the experiments one of the two inputs was, before drug application, repeatedly tetanized to induce LTP, causing a $100-200 \%$ (mean value $=149 \%$ ) potentiation. The subsequent application of DPCPX increased both inputs to an equal degree (paired difference of $2.3 \pm 11.3 \%, n$ $=4$ ). When tetanized in the presence of DPCPX no LTP was observed in the previously tetanized inputs $(-4.1 \%)$ compared to an LTP of $46 \%$ in the naive ones (measured $10 \mathrm{~min}$ after the tetanus, $n=4$ ), that is, indicating occlusion between LTPs evoked at these different conditions of release probability.

\section{Time course of LTP in the presence of adenosine agonist/antagonists}

Single 10 impulse tetani were applied in the presence of $\mathrm{CHA}$ (100-200 nM), in the presence of DPCPX (200 nM), and in the absence of both drugs, and the resulting LTP time courses were compared. The average magnitude of the field EPSPs before tetanization did not differ between these three groups $(0.10 \pm .01$ $\mathrm{mV} / \mathrm{msec}$ for all three groups, after adjustment of stimulus strength; see Materials and Methods). Figure 1 shows the average growth phase of LTP obtained without drugs $(A, n=34)$, in the presence of CHA $(B, n=24)$, and in the presence of DPCPX $(C, n=10)$, respectively. These curves demonstrate that the change in release probability induced by these drugs, indicated by an almost tenfold change in field EPSP magnitude, had little influence on the magnitude and time course of the growth phase of LTP (see also Table 2). However, comparison 
Table 2. Peak value and decay characteristics of LTP obtained in "control" solution in the presence of CHA and in the presence of DPCPX, respectively

\begin{tabular}{llll} 
& CHA & "Control" & DPCPX \\
\hline Peak & $125 \pm 7.8 \%$ & $122 \pm 9.4 \%$ & $109 \pm 5.4 \%$ \\
& $n-24$ & $n=34$ & $n=10$ \\
Decay; peak to 3 min & $46.3 \pm 2.05 \%$ & $27.9 \pm 1.8 \%$ & $16.6 \pm 3.4 \%$ \\
& $n=24$ & $n=34$ & $n=10$ \\
Decay; 3 min to 30 min & $56.2 \pm 3.6 \%$ & $52.3 \pm 3.4 \%$ & $51.4 \pm 7.6 \%$ \\
& $n=24$ & $n=34$ & $n=10$ \\
Decay; 30 min to 55 min & $12.4 \pm 15.3 \%$ & & $17.3 \pm 16.8 \%$ \\
& $n=5$ & & $n=5$
\end{tabular}

Values were calculated as described in the legend to Figure 3.

between the curves indicates that the subsequent decay of LTP was somewhat affected by the release changes - the lower release probability in CHA $(B)$ leading to more decay.

Figure 2 shows the same curves as in Figure 1 but on a longer time scale, demonstrating that the adenosine agonist/antagonists did not change the overall LTP time course. However, as also indicated in Figure 1, the LTP decay was more pronounced in the presence of CHA $\left(A_{2}\right)$ leading to a significantly smaller "steady state" value. Figure $2 B$, in which the curves obtained in CHA (Fig. 2 $A_{2}$ ) and DPCPX $\left(A_{3}\right)$, respectively, are shown superimposed, indicates a near parallel displacement of the LTP curves suggesting a difference in decay only relating to the first few minutes after the peak. This indication is also borne out by the comparison between the early (peak, $3 \mathrm{~min}$ ) and later (3$30 \mathrm{~min}$ ) decays of the LTPs (Table 2).

\section{Effect of different induction conditions on the decay of LTP}

To examine whether the change in LTP time course observed after $\mathrm{CHA}$ application could be related to variations in induction
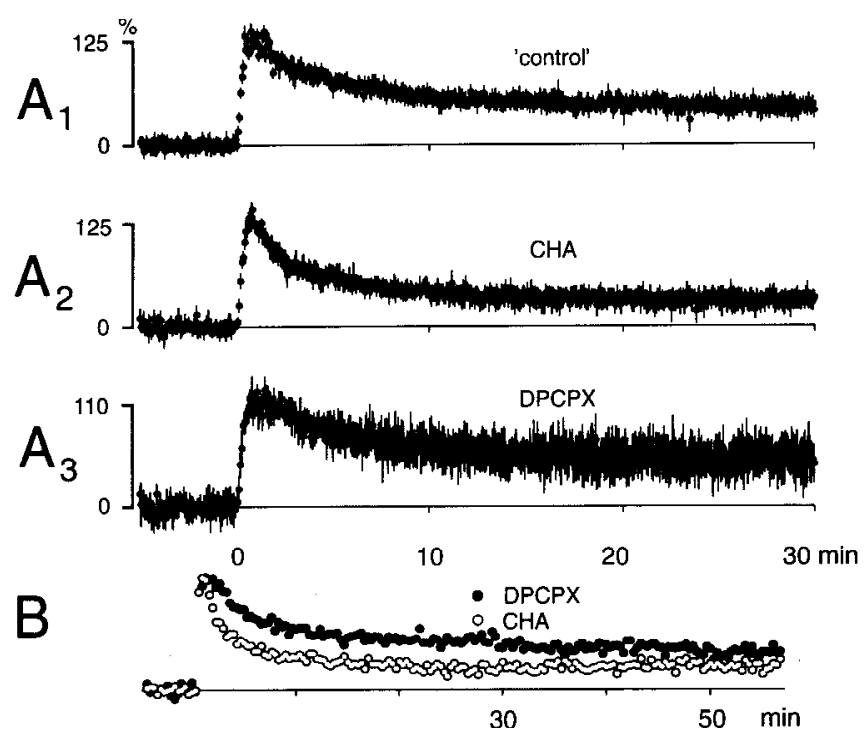

Figure 2. LTP time course following a 10 impulse tetanus $(50 \mathrm{~Hz})$ in different conditions of release probability. $A_{1}-A_{3}$, Same curves as in Figure $1 A-C$, respectively, but shown with a longer time scale. $B$, LTP time course following a 10 impulse tetanus in the presence of $\mathrm{CHA}$ (open circles, $n=5$ ) and DPCPX (solid circles, $n=5$ ) followed for up to $60 \mathrm{~min}$. Before averaging of the potentiation curves, every $20 \mathrm{sec}$ segment (five values) was replaced by the average value for those values. In the figure the potentiations are normalized to a common peak amplitude. conditions following drug application, it was first tested whether the LTP time course was related to the amount of LTP produced by a given tetanus (cf. Malenka, 1991; Hanse and Gustafsson, 1992 ). Figure 3 shows that both the early $(A)$ and later $(B)$ decay of LTP (see above) were independent of the absolute value of potentiation reached at the peak of LTP, both without any drug
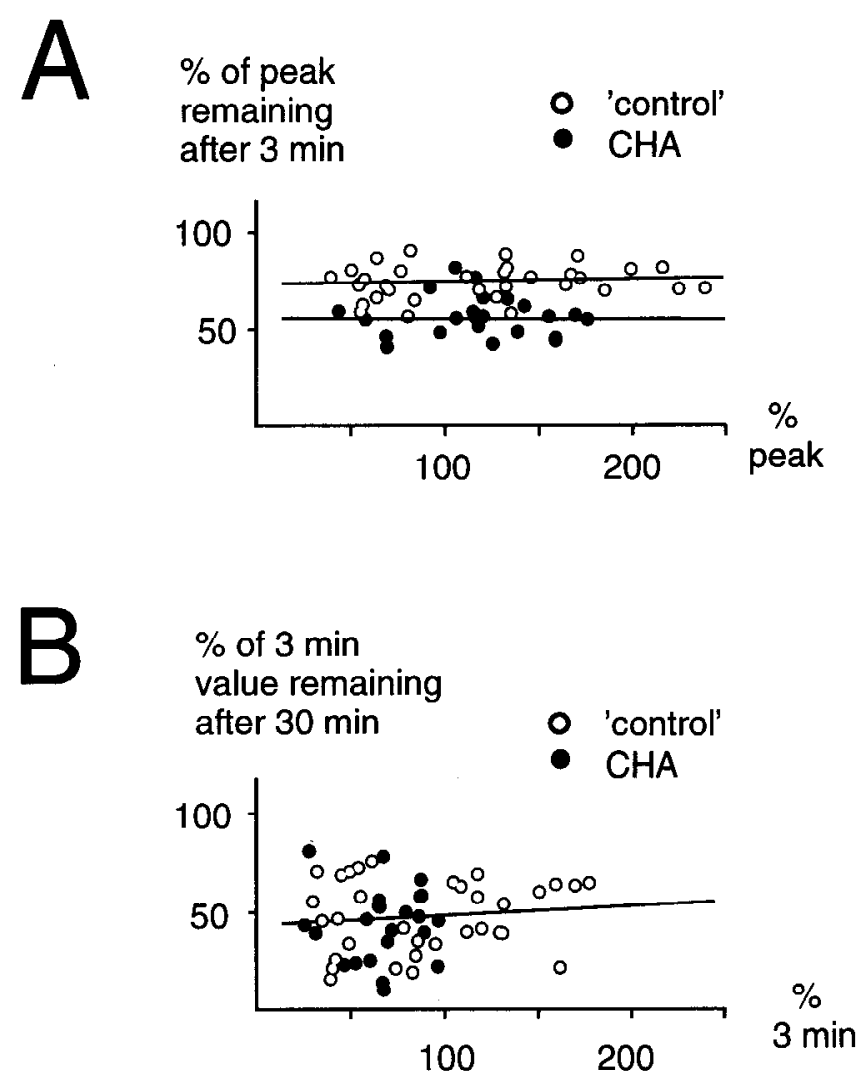

Figure 3. Relation between amounts of LTP measured at different time points after the induction. $A$, Relation between the peak value, and the fraction of this peak value remaining at $3 \mathrm{~min}$ after LTP induction, of LTPs induced by a 10 impulse tetanus in the "control" solution (solid circles) and in the presence of CHA (open circles). The peak value was calculated as the average of measurements taken between 30 and $60 \mathrm{sec}$ following the tetanus. The $3 \mathrm{~min}$ value was calculated as the average of 15 measurements $(60 \mathrm{sec})$ surrounding that time point. To test a null hypothesis that the regression for the "control" and CHA cases were identical, we used an $F$ test of a reduced model (all data pooled for the regression) versus a full model (multiple linear regression using dummy coding for the CHA treatment). The full model yielded a better fit, which was significant at the $p<0.01$ level. $B$, Relation between the potentiation observed $3 \mathrm{~min}$ after LTP induction and the fraction of this value remaining after $30 \mathrm{~min}$ : 

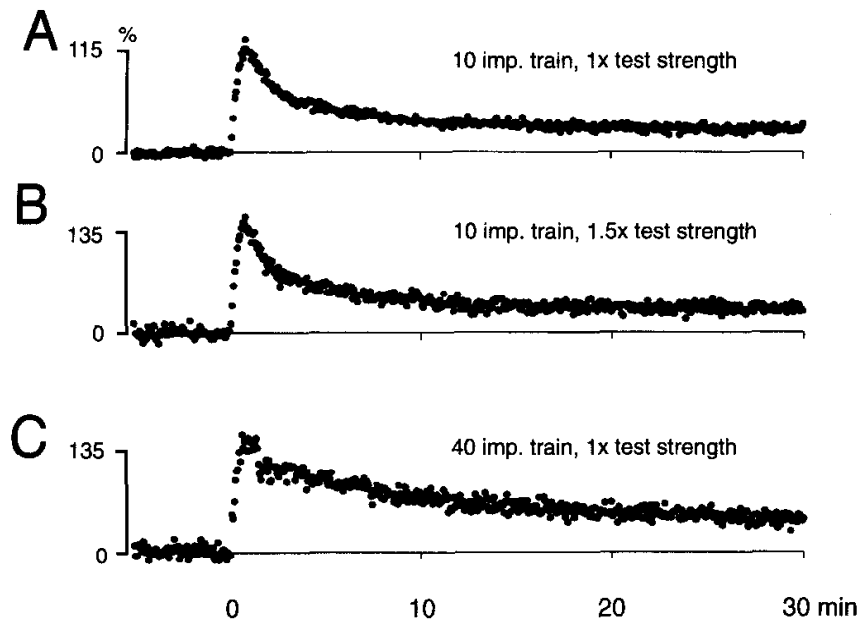

Figure 4. LTP time course following a brief tetanus of different duration and strength in the presence of CHA. $A$, Average EPSP changes ( + SEM), as percentage of the average EPSP value obtained during the $60 \mathrm{sec}$ immediately preceding the tetanus, observed after a 10 impulse tetanus evoked at $1 \times$ test strength $(n=10)$. To obtain the early time course exclusive of augmentation, the original potentiation values were divided by the averaged augmentation curve obtained in the same experiments in the presence of D-AP5. All potentiations were normalized before averaging to a common peak amplitude. $B$, Same as in $A$, but the tetanus was evoked at $1.5 \times$ test strength $(n=14)$. $C$, Same as in $A$, but the tetanus consisted of 40 impulses evoked at $1 \times$ test strength.

(open circles) and in the presence of CHA (solid circles). LTP was also (in the presence of CHA) evoked by a stronger tetanus (10 impulse tetanus using $1.5 \times$ the test intensity), leading to a similarly decaying potentiation (compare Fig. $4, A$ and $B$ ). On the other hand, following a longer (40 impulse) tetanus (in the presence of CHA) delivered at test intensity, the early decay became less $(20.6 \pm 3.3 \%$; see Table 2$)$.

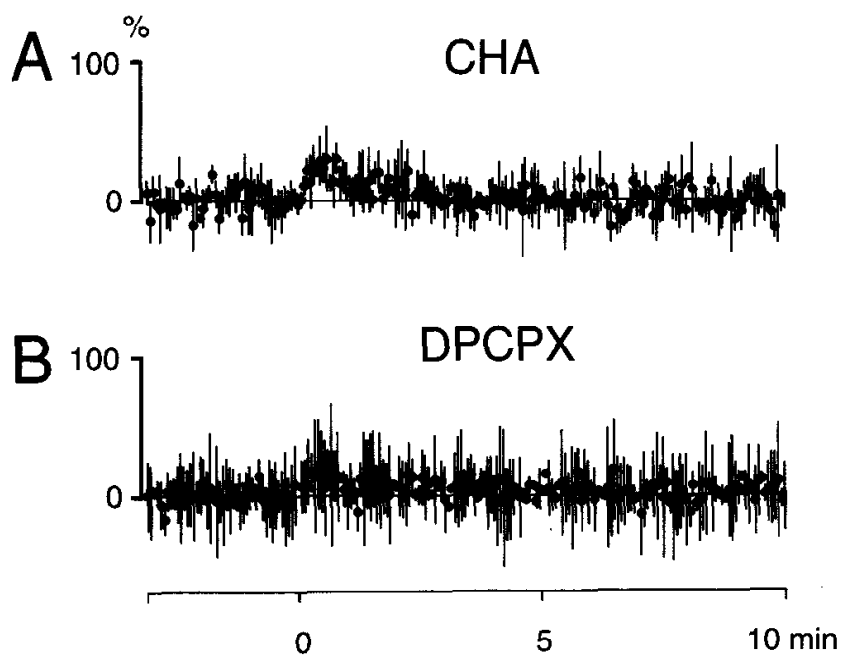

Figure 5. Occlusion of LTP by prior tetanization in CHA and in DPCPX. $A$, In the presence of CHA (200 nM): averaged values ( \pm SEM) of the potentiation given by a 10 impulse tetanus after division by augmentation. Prior to this tetanus the input had been repeatedly tetanized (see Materials and Methods) in order to saturate LTP (last tetanus was applied $45 \mathrm{~min}$ prior to the 10 impulse tetanus) and the stimulus intensity was adjusted to obtain an EPSP of similar magnitude as that prior to the saturating tetanization. $B$, Same as in $A$, but in the presence of $200 \mathrm{~nm}$ DPCPX.
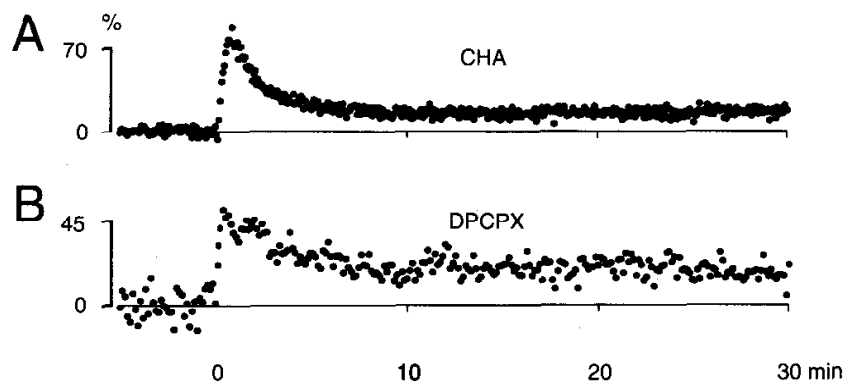

Figure 6. Potentiation of the NMDA receptor-mediated EPSP, induced by a 10 impulse tetanus. $A$, Average EPSP changes, as percentage of the average EPSP value obtained during the $60 \mathrm{sec}$ immediately preceding the tetanus, in the presence of $\mathrm{CHA}(n=8)$. To obtain the early time course exclusive of augmentation, the original potentiation values were divided by the averaged augmentation curve obtained in the experiments shown in Figures 1 and 2. B, Same as in $A$ but in the presence of DPCPX.

\section{Occlusion of LTP by prior tetanization, in CHA and in $D P C P X$}

Following repeated tetanization a single 10 impulse tetanus evokes little or no potentiation (except augmentation) when tested one hour afterwards (Gustafsson et al., 1989), indicating an occlusion between the early transient and the more sustained phase of LTP. Figure 5 shows that this was also the case in the presence of CHA $(A)$ or of DPCPX $(B)$, the single 10 impulse tetanus being elicited $45 \mathrm{~min}$ after the last saturating tetanus. As described in the Materials and Methods, the inputs had been subjected to repeated tetanization until no further LTP could be evoked, this procedure generating a potentiation of $174 \pm$ $12 \%(n=4)$ and $192 \pm 24 \%(n=4)$ in the presence of CHA and DPCPX, respectively, measured 15-20 min after the last tetanus. This latter result also demonstrates that the maximal amount of LTP that can be obtained was much the same in the presence of CHA or DPCPX.

\section{Potentiation of the NMDA receptor-mediated EPSP in CHA and in $D P C P X$}

In the presence of the non-NMDA receptor antagonist CNQX $(10 \mu \mathrm{M})$ and low $(0.1 \mathrm{~mm})$-magnesium solution, NMDA-mediated EPSPs can be observed in isolation (Andreasen et al., 1989). Following a single 10 impulse tetanus this NMDA EPSP shows an LTP with similar time characteristics as that of the non-NMDA EPSP (Asztely et al., 1992). Figure 6 shows such LTPs of the NMDA EPSP in the presence of CHA $(A)$ and DPCPX $(B)$, respectively, and demonstrates that the time course of the NMDA EPSP LTP was affected by adenosine agonist/ antagonists in the same manner as the non-NMDA EPSP LTP (compare with Fig. 2). The peak magnitude of the NMDA EPSP LTP was $70 \pm 11.9 \%(n=8)$ and $43 \pm 5.1 \%(n=5)$ in CHA and in DPCPX, respectively, that is, smaller than the peak potentiation of the non-NMDA EPSP LTP (see Table 2).

\section{Potentiation of combined non-NMDA/NMDA EPSPS in the} presence of $\mathrm{CHA}$

The larger peak magnitude of the NMDA EPSP LTP in the presence of CHA, compared to that in the presence of DPCPX, might be taken to suggest that CHA leads to an increase in the NMDA/non-NMDA EPSP LTP ratio. Asztely et al. (1992) demonstrated, using lower CNQX ( $1 \mu \mathrm{M})$ to allow for the measurement of non-NMDA and NMDA EPSP potentiation in parallel 


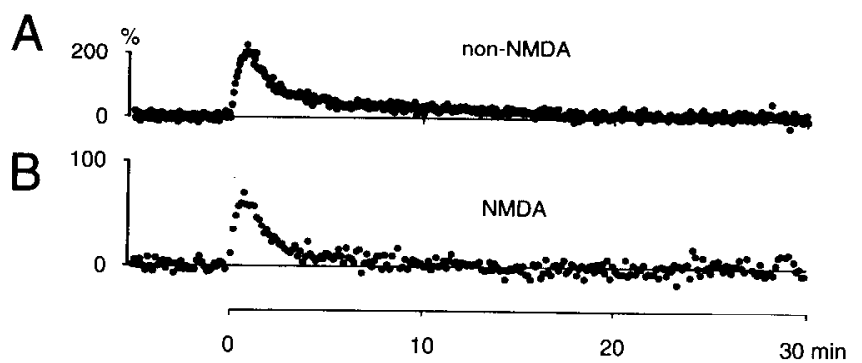

Figure 7. Potentiation of field EPSPs in low magnesium $(0.1 \mathrm{~mm})$ and CNQX $(1.0 \mu \mathrm{M})$, induced by a 10 impulse tetanus. In this solution the field EPSP consisted of a non-NMDA and a NMDA component of comparable magnitudes, which could be measured simultaneously (cf. Asztely et al., 1992). $A$, Average non-NMDA component changes, as percentage of the average valuc obtained during the $60 \mathrm{sec}$ immediately preceding the tetanus, in the presence of CHA $(n=5)$. To obtain the early time course exclusive of augmentation, the original potentiation values were divided by the averaged augmentation curve obtained in the experiments shown in Figures 1 and 2. $B$, Same as in $A$ but for the NMDA component of the EPSP. The curve has been scaled to match the peak amplitude of the curve of the non-NMDA potentiation (note the different scales of the ordinates).

(see also Materials and Methods), that the NMDA EPSP LTP had a magnitude about one-third of that of the non-NMDA EPSP LTP. Figure 7 shows such parallel measurements of the potentiation (exclusive of augmentation) of the non-NMDA EPSP $(A)$ and NMDA EPSP $(B)$ following single 10 impulse tetani in the presence of $\mathrm{CHA}$, the scale on the ordinate in $B$ being expanded three times compared to that in $A$. As indicated in the figure, no stable potentiation of either the non-NMDA EPSP or NMDA EPSP could be elicited in this solution, possibly due to an impaired induction under these circumstances (cf. Malenka, 1991; Hanse and Gustafsson, 1992). Nevertheless, the similar size of the potentiations indicates an approximate $1: 3$ ratio of NMDA/non-NMDA EPSP LTP also in the presence of CHA.

\section{Time course of LTP using different calcium/magnesium ratios}

Changes in extracellular calcium/magnesium concentration ratio was also used to alter the release probability (Malinow and Tsien, 1990; Otmakhov et al., 1993). A decrease/increase in the extracellular calcium/magnesium concentration to $2 / 6 \mathrm{~mm}$ increased the paired-pulse facilitation to $106 \pm 18.5 \%(n=5)$, indicating a change in release probability in the same range as with application of CHA. Figure $8 A$ shows that LTP obtained in this solution exhibits a time course similar to that obtained in the presence of CHA (see also Table 3). In other experiments release probability was increased by lowering extracellular magnesium, giving a 4/2 mM calcium/magnesium solution. The LTP time course obtained in this solution resembled that seen in the presence of DPCPX (Fig. 8B; see also Table 3).

\section{Discussion}

A recent suggestion is that the expression of LTP in the hippocampal CAl region is both pre- and postsynaptically located, and that the relative contribution of these locations depends on the synaptic release probability (Larkman et al., 1992; Liao et al., 1992). The objective of the present study was to find, albeit in an indirect manner, evidence for this notion by examining some LTP characteristics after changes in transmitter release induced by adenosine agonist/antagonists. The main finding in

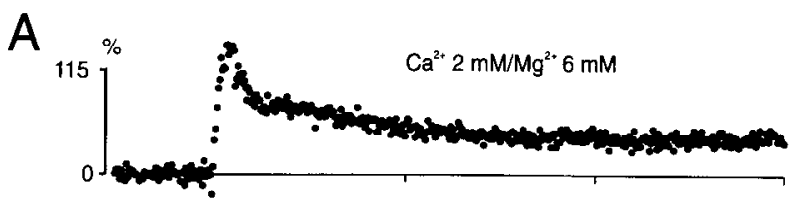

B

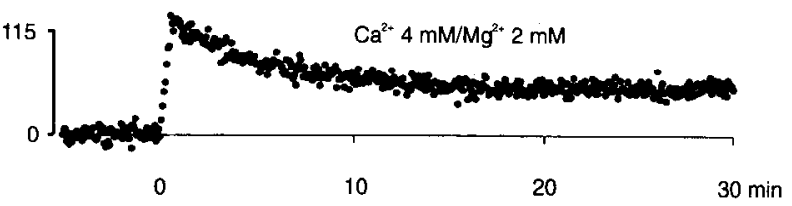

Figure 8. LTP time course following a 10 impulse tetanus in conditions of different extracellular calcium/magnesium ratios. $A$, Averaged EPSP changes, as percentage of the averaged EPSP value obtained during the $60 \mathrm{sec}$ immediately preceding the tetanus, in $2 \mathrm{~mm}$ calcium and $6 \mathrm{~mm}$ magnesium. To obtain the early time course exclusive of augmentation, the original potentiation values were divided by the averaged augmentation curve obtained in the same experiments in the presence of D-AP5. $B$, Same as in $A$, but in $4 \mathrm{~mm}$ calcium and $2 \mathrm{~mm}$ magnesium.

the present study was that these LTP characteristics, including overall LTP time course (onset latency, growth phase, and subsequent decay for both non-NMDA and NMDA EPSPs) following a brief tetanus, and the relative ratio between the LTPs of the non-NMDA and NMDA EPSPs, were essentially the same over an almost 10 -fold range in release probability (measured as change in EPSP magnitude). It was also observed that LTP evoked at one level of release probability occluded that induced at a lower (or higher) level, and that induced changes in the release probability affected inputs with established LTP and "naive" inputs to an equal extent. Thus, these data appear to offer no support for the notion of different locations for LTP expression at different conditions of release probability.

\section{The use of adenosine agonist/antagonists to modulate release} probability

In the present study adenosine $\mathrm{Al}$ receptor agonist/antagonist were the main agents used to modulate the release probability. The release probability differs between individual synapses in hippocampal slices (Larkman et al., 1992; Liao et al., 1992). In the study by Larkman et al. (1992) changes in extracellular calcium/magnesium were used to vary the initial release probability. It was presently assumed that adenosine would also affect the synapses, and give an overall lowering of release probability (Lupica et al., 1992; Prince and Stevens, 1992; Scholz and Miller, 1992) similar to that given by changes in extracellular calcium/magnesium (Malinow and Tsien, 1990; Otmakhov et al., 1993). A recent study of autaptic synapses has, how-

Table 3. Peak value and decay of LTP obtained using different calcium/magnesium concentration ratios

\begin{tabular}{lll} 
& {$\left[\mathrm{Ca}^{2+}\right]_{\mathrm{EC}} 2 \mathrm{mM}$,} & {$\left[\mathrm{Ca}^{2+}\right]_{\mathrm{EC}} 4 \mathrm{mM}$,} \\
{$\left[\mathrm{Mg}^{2+}\right]_{\mathrm{EC}} 6 \mathrm{mM}$} & {$\left[\mathrm{Mg}^{2+}\right]_{\mathrm{EC}} 2 \mathrm{mM}$} \\
\hline Peak & $117 \pm 7.7 \%$ & $115 \pm 9.6 \%$ \\
& $n=5$ & $n=6$ \\
Decay; peak to $3 \mathrm{~min}$ & $44.2 \pm 5.4 \%$ & $21.9 \pm 1.9 \%$ \\
& $n=5$ & $n=6$ \\
Decay; 3 min to 30 min & $44.8 \pm 5.5 \%$ & $47.9 \pm 2.5 \%$ \\
& $n=5$ & $n=6$ \\
\hline
\end{tabular}


ever, shown that baclofen, in contrast to calcium/magnesium changes, affects presynaptic release in a nonuniform manner, having less effect on high- than on low release sites (Rosenmund et al., 1993). If this would be true also for adenosine in the slice preparation, it would complicate the interpretation of data obtained using adenosine agonist/antagonists (see below). However, certain key findings (see below) obtained using these drugs were also obtained when changing release by altering the calcium/magnesium ratio.

\section{$L T P$ of the non-NMDA receptor-mediated EPSP}

The notion that LTP in the CAl region, even within the first hour or so, is not based on a single expression mechanism has been on the agenda for some time. Some studies have suggested that these different expression mechanisms are temporally separate, for example, an early transient presynaptic expression followed by a later, more stable, postsynaptic one (Davies et al., 1989; Reymann et al., 1990), a subdivision in line with the notion of an early decremental, short-term potentiation (STP), and a later, longer-lasting, nondecremental (LTP) component of NMDA dependent potentiation (Kennedy, 1988; Bliss and Collingridge, 1992). The study of Larkman et al. (1992) did not indicate such strict temporal separation, but a rapid onset of both the presumed pre- and postsynaptic modifications. Nevertheless, their results suggested differences in time course for the two modifications, with a more transient character of the postsynaptic modification, and a nondeclining nature of the presynaptic one. Thus, changes in the release probability (prior to tetanization) might be expected to lead to changes in the LTP time course. However, in the present study the LTP time course was essentially the same at the different levels of release probability, with a latency of less than $4 \mathrm{sec}$, and a 30-40 sec growth phase followed by a subsequent decay, the only observed difference being a greater decay during the first $3 \mathrm{~min}$ for LTP evoked at low release probability.

\section{$L T P$ of the NMDA receptor-mediated EPSP}

Recent studies have shown that the NMDA EPSP also undergoes LTP (Bashir et al., 1991; Berretta et al., 1991; Asztely et al., 1992), with a time course quite similar to that of the nonNMDA EPSP LTP, but quantitatively less, only about one-third of that of the latter LTP (Asztely et al., 1992). Nevertheless, such an NMDA EPSP LTP may be suggestive of a presynaptic component of LTP, and, if so, its magnitude might vary strongly with changes in release probability. Although the NMDA EPSP LTP was found to be less in DPCPX than in CHA, even in DPCPX a considerable NMDA EPSP LTP could be induced, with a peak potentiation about $60 \%$ of that obtained in $\mathrm{CHA}$. Moreover, the time course of the NMDA EPSP LTP was affected by alterations in release probability in the same manner as the non-NMDA EPSP LTP, showing a faster early decay in CHA than in DPCPX. Finally, the NMDA/non-NMDA EPSP LTP ratio had the same quantitative relation in $\mathrm{CHA}$ as has been previously described in the absence of this drug (Asztely ct al., 1992).

\section{LTP decay at different release probabilities}

As noted above, LTPs evoked under conditions of low release probability displayed a somewhat greater decay during the first few minutes. The question then arises whether this discrepancy could be a consequence of changes in LTP at a single site with altered release, for example, in its induction, or reflect a change in location for LTP expression with different levels of release probability. With respect to the first alternative, the initial decay of LTP was found to be little affected by variations in induction strength (and amount of LTP produced) for a given tetanus length, both in the control material and that obtained in the low-release situation. It seems thus unlikely that the faster decay observed in the low-release condition should be due to less postsynaptic activation under these conditions. On the other hand, prolongation of the afferent spike train generated an LTP with an initial decay that was similar to that obtained by a 10 impulse tetanus in control solution. This result may suggest that the low-release condition impairs some induction factor that can be overcome with prolongation of the afferent spike train, and that the discrepancy in initial decay in the different release conditions may be due to an induction factor (cf. Mitchell et al., 1993).

If, on the other hand, the LTPs observed at the different levels of release probability represent expression at two different locations, the present data suggest that the underlying mechanisms at these two locations should operate with very similar time characteristics. Since the variation in initial decay was also observed for the NMDA EPSP LTP, one would have to assume that this LTP should also change location for its expression with altered release, and, as indicated by the present results, keep its relative magnitude to that of the non-NMDA EPSP LTP. Moreover, the difference in LTP time course at different levels of release probability presently observed does not resemble the one indicated by the results of Larkman et al. (1992) with a more transient character of the postsynaptic modification (present at levels of high release) and a nondeclining nature of the presynaptic one (present at low release).

The faster initial decay of LTP in the low-release situation might be compatible with LTPs with considerably different time characteristics pre-and postsynaptically. The assumption would then be that the population of synapses examined consists of two groups, one with a high release probability that is more or less unaffected by adenosine, and a second group with low release probability that is affected by adenosine (compare baclofen above). Thus, in CHA the further lowering of release probability might impair the induction (following a brief tetanus) of a presynaptically located LTP in the low-release group, while leaving the induction of a postsynaptically located LTP in the highrelease group unaffected. If this latter LTP is more transient than the LTP in the low-release group, as indicated by the results by Larkman et al. (1992), the initial decay would be faster in the presence of CHA. However, changes in the extracellular calcium/magnesium ratio that should produce a uniform change in release probability (Rosenmund et al., 1993), affected the initial decay of LTP in the same manner as adenosine Al agonist/antagonists. Moreover, prior repeated tetanization in the presence of CHA or DPCPX, respectively, occluded also the early part of LTP induced by a brief tetanus, indicating that the samc mechanism(s) undcrlies both the latcr (stablc) phase of LTP and the early (transient) phase at both levels of release.

\section{Occlusion between LTPs evoked at different release probabilities}

In the present study LTP evoked at saturated amounts at one level of release probability fully occluded the generation of LTP at another level. In addition, adenosine agonist/antagonists, when 
applied on an EPSP saturated with respect to LTP, affected this EPSP quantitatively the same as that of a naive input, a result previously also observed with respect to changes in extracellular calcium/magnesium (Huang et al., 1987, 1988). These results are most easily explained by the same location(s) for LTP expression at both levels of release. To account for different expression locations at the different levels of release probability the generation of LTP at one location should have to occlude induction/expression of LTP at the other location, or LTP should always be generated at both locations, but expressed in one or the other location dependent on the level of release probability. The location for LTP expression should also be unchanged if release probability is changed after induction of LTP, or the conversion of LTP from one location to the other during the change in release probability should take place in a strictly inversely proportional manner.

\section{Effect of adenosine on LTP per se}

Several groups have reported that the addition of adenosine or adenosine agonist block LTP in hippocampal slices in vitro (Arai et al., 1990; de Mendonca and Ribeiro, 1990). However, in a recent study (Mitchell et al., 1993) such a block was not observed (see also Dunwiddie and Hoffer 1980). According to Mitchell et al. (1993) the inhibitory effect of adenosine on LTP should be indirect via a decreased transmitter release at individual excitatory synapses, leading to a decreased postsynaptic response during the high-frequency stimulation inducing LTP. In the present study LTP was easily induced in the presence of an adenosine agonist, in line with the result by Mitchell et al. (1993). A possible reason for the easy induction of LTP could have been the use of the $\mathrm{GABA}_{\mathrm{A}}$ antagonist picrotoxin, removing the increased inhibitory influence otherwise obtained in the presence of an adenosine agonist (Lambert and Teyler, 1991).

\section{Conclusion}

The present results have shown that the carly time course of LTP (of both the non-NMDA and NMDA receptor-mediated EPSPs) is essentially unaltered by changes in release probability induced by adenosine agonist/antagonists. When applied on established LTP these drugs also affect the potentiated EPSPs to the same degree as EPSPs evoked by "naive" inputs. The results do not provide evidence favoring the notion of different locations of the LTP expression mechanisms at different levels of release probability.

\section{References}

Andreasen M, Lambert J, Skovgaard Jensen M (1989) Effects of new non- $N$-methyl-D-aspartate antagonists on synaptic transmission in the in vitro rat hippocampus. J Physiol (Lond) 414:317-336.

Arai A, Kessler M, Lynch G (1990) The effect of adenosine on the development of long-term potentiation. Neurosci Lett 119:41-44.

Asztely F, Wigström H, Gustafsson B (1992) The relative contribution of NMDA receptor channels in the expression of long-term potentiation in the hippocampal CA1 region. Eur J Neurosci 4:681-690.

Bashir Z, Alford S, Davies S, Randall A, Collingridge G (1991) Longterm potentiation of NMDA receptor-mediated synaptic transmission in the hippocampus. Nature 349:156-158.

Berretta N, Berton F, Bianchi R, Brunelli M, Capogna M, Francesconi W (1991) Long-term potentiation of NMDA receptor-mediated EPSP in guinea-pig hippocampal slices. Eur J Neurosci 3:850-854.

Bliss TVP, Collingridge $G$ (1993) A synaptic model of memory: longterm potentiation in the hippocampus. Nature 361:31-39.

Davies S, Lester R, Reymann K, Collingridge G (1989) Temporally distinct pre- and postsynaptic mechanisms maintain long-term potentiation. Nature 338:500-503.

de Mendonca A, Ribeiro J (1990) 2-Chloroadenosine decreases longterm potentiation in the hippocampal CA1 area of the rat. Neurosci Lett 118:107-111.

Dunwiddie T, Hoffer B (1980) Adenine nucleotides and synaptic transmission in the in vitro rat hippocampus. Br J Pharmacol 69:59-68.

Dunwiddie T, Miller K (1993) Effects of adenosine and cadmium on presynaptic fiber spikes in the CA1 region of rat hippocampus in vitro. Neuropharmacology 32:1061-1068.

Gustafsson B, Asztely F, Hanse E, Wigström H (1989) Onset characteristics of long-term potentiation in the guinea-pig hippocampal CA1 region in vitro. Eur J Neurosci 1:382-394.

Hannay T, Larkman A, Stratford K, Jack J (1993) A common rule governs the synaptic locus of both short-term and long-term potentiation. Curr Biol 3:832-841.

Hanse E, Gustafsson B (1992) Postsynaptic, but not presynaptic, activity controls the early time course of long-term potentiation in the dentate gyrus. J Neurosci 12:3226-3240.

Huang Y-Y, Wigström H, Gustafsson B (1987) Facilitated induction of hippocampal long-term potentiation in slices perfused with low concentrations of magnesium. Neuroscience 23:9-16

Huang Y-Y, Gustafsson B, Wigström H (1988) Facilitation of hippocampal long-term potentiation in slices with high concentrations of calcium. Brain Res 456:88-94.

Kennedy MB (1988) Synaptic memory molecules. Nature 335:770772.

Kullman D, Nicoll R (1992) Long-term potentiation is associated with increases in quantal content and quantal amplitude. Nature 357:240244.

Lambert N, Teyler T (1991) Adenosine depresses excitatory but not fast inhibitory synaptic transmission in area $\mathrm{CAl}$ of the rat hippocampus. Neurosci Lett 122:50-52.

Larkman A, Hannay T, Stratford K, Jack J (1992) Presynaptic release probability influences the locus of long-term potentiation. Nature 360 : $70-73$.

Liao D, Jones A, Malinow R (1992) Direct measurements of quantal. changes underlying long-term potentiation in CAl hippocampus.' Neuron 9:1089-1097.

Lupica C, Proctor W, Dunwiddie T (1992) Presynaptic inhibition of excitatory synaptic transmission by adenosine in rat hippocampus: analysis of unitary EPSP variance measured by whole-cell recording. J Neurosci 12:3753-3764.

Magleby K, Zengel J (1976) Augmentation: a process that acts to increase transmitter release at the frog neuromuscular junction. $J$ Physiol (Lond) 257:449-470.

Malenka R (1991) Postsynaptic factors control the duration of synaptic enhancement in the area CA1 of the hippocampus. Neuron 6:53-60.

Malenka R, Lancaster B, Zucker R (1992) Temporal limits on the rise in postsynaptic calcium required for the induction of long-term potentiation. Neuron 9:121-128.

Malinow R, Tsien R (1990) Presynaptic enhancement shown by wholecell recordings of long-term potentiation in hippocampal slices. $\mathrm{Na}$ ture 346:177-180.

McNaughton BL (1982) Long-term enhancement and short-term potentiation in rat fascia dentata act through different mechanisms. J Physiol (Lond) 324:240-262.

Mitchell J, Miller K, Dunwiddie T (1993) Adenosine-induced suppression of synaptic responses and the initiation and expression of long-term potentiation in the CAl region of the hippocampus. Hippocampus 3:77-86.

Otmakhov N, Shirke A, Malinow R (1993) Measuring the impact of probabilistic transmission on neuronal output. Neuron 10:1101-1111.

Prince D, Stevens C (1992) Adenosine decreases neurotransmitter release at central synapses. Proc Natl Acad Sci USA 89:8586-8590.

Reymann K, Davies S, Matthies H, Kase H, Collingridge G (1990) Activation of a K-252b-sensitive protein kinase is necessary for a post-synaptic phase of long-term potentiation. Eur J Neurosci 2:481486.

Rosenmund C, Clements J, Westbrook G (1993) Nonuniform probability of glutamate release at a hippocampal synapse. Science 262 : 754-757.

Scholz K, Miller R (1992) Inhibition of quantal transmitter release in 
the absence of calcium influx by a $\mathrm{G}$ protein-linked adenosine receptor at hippocampal synapses. Neuron 8:1139-1150.

Thompson S, Haas H, Gäwihler B (1992) Comparison of the actions of adenosine at pre- and postsynaptic receptors in the rat hippocampus in vitro. J Physiol (Lond) 451:347-363.
Wigström H, Gustafsson B (1985) Facilitation of hippocampal longlasting potentiation by GABA antagonists. Acta Physiol Scand 125 159-172.

Zucker RS (1989) Short-term synaptic plasticity. Annu Rev Neurosci $12: 13-31$. 